Electronic properties of nanotube-based sensors: An inverse modeling approach

This content has been downloaded from IOPscience. Please scroll down to see the full text. 2008 EPL 8227004

(http://iopscience.iop.org/0295-5075/82/2/27004)

View the table of contents for this issue, or go to the journal homepage for more

Download details:

IP Address: 134.226.254.162

This content was downloaded on 28/01/2014 at 09:01

Please note that terms and conditions apply. 


\title{
Electronic properties of nanotube-based sensors: An inverse modeling approach
}

\author{
C. G. RochA ${ }^{(a)}$, A. WAll and M. S. Ferreira ${ }^{(b)}$ \\ School of Physics, Trinity College Dublin - Dublin 2, Ireland, EU
}

received 4 January 2008; accepted in final form 5 March 2008

published online 11 April 2008

PACS 71.15.-m - Methods of electronic structure calculations

PACS 73.22.-f - Electronic structure of nanoscale materials: clusters, nanoparticles, nanotubes, and nanocrystals

PACS 71.55.-i - Impurity and defect levels

\begin{abstract}
Nanotube-based sensors depend on significant conductivity changes induced by doping. Predictions of which impurity/nanotube combination provides efficient sensor characteristics are usually made on a case-by-case basis, following the study of how a particular nanotube responds to the presence of a specific doping agent. With a multitude of possible combinations, this so-called forward modeling approach is unable to address questions of general nature, like, for instance, the necessary features the components must have to produce certain physical properties on the device. Questions of this nature call for an inverse modeling scheme in which information about the sensor components can be extracted from the knowledge of a few physical quantities demanded for the device. Here we make use of a mathematically transparent formalism that works in both the forward and the inverse directions. We argue that this method can provide general guidelines on the absorption process and narrow the search for the ideal combination of tube and doping agents required to produce efficient nanoscopic sensors.
\end{abstract}

Copyright (C) EPLA, 2008

One remarkable feature of carbon nanotubes (NT) is that their conductance is affected by the interaction with certain foreign objects (FO). Atoms, molecules and nanoparticles are some of the objects known to interact strongly with NT, paving their way to being used as nanoscopic sensors [1-4]. From the theoretical point of view, the problem of which FO produces a good NT-based sensor involves two separate studies: one addressing the conditions under which the FO adheres to the NT, and another investigating the effect that this interaction brings to the electronic properties of the device.

The first stage of the calculation is usually performed by $a b$ initio evaluations providing the energetic cost of doping a NT with a single FO. Such a study indicates the binding energy between tube and FO, including the most favourable location for adhesion and the amount of charge exchanged between them. The second consists of evaluating the conductance of the NT in the presence of a finite concentration of objects. In this case, dealing with a single doping agent is insufficient given that the conductance is sensitive to how disordered the scatterers

\footnotetext{
(a) E-mail: gomesdac@tcd.ie

(b) E-mail: ferreirm@tcd.ie
}

are. This requires averaging over a large number of configurations, making the ab initio approach unsuitable due to its large computational cost. To overcome this limitation the latter stage of the calculation is usually done by using less computationally intensive semi-empirical Hamiltonians whose parameters are fitted to the electronic structure of a singly doped NT $[5,6]$.

It is unfortunate that both stages of the calculation are performed separately and disconnectedly since the lack of transparency involved in the numerical intricacies of both stages conceals the general role played by each part involved in the interaction. For a given set of results, one can hardly distinguish the separate contributions coming from the NT and from the FO. Therefore, it is very difficult to make general statements about the interaction process between tube and doping agents and predictions are on a case-by-case basis. In fact, NT doped with several atomic impurities were surveyed and values for their respective binding energies, equilibrium positions and magnetic moments were reported [7]. Although overall trends can be identified in such studies, they are not able to address questions of more general nature. For instance, for a given doping agent, how will the reported values depend on the NT features such as diameter and 
chirality? Or for a given tube, what characteristics are required in the doping FO if they are to maximally affect the NT conducting properties? These questions cannot be answered by ad hoc strategies and require an inverse modeling approach. Rather than calculating the effect that a specific NT/FO pair will have on the physical properties of the system, also known as forward modeling, the inverse scheme consists of extracting information about the pair components from the knowledge of certain physical quantities. Such a scheme is essential for establishing general guidelines on the search for ideal NT-based sensors.

It is the goal of this manuscript to present a method capable of bridging the gap between the aforementioned calculation stages so that we can establish a more transparent way of studying the effect of doping on the physical properties of NT. In particular, it is our aim to introduce a scheme that allows one to identify separate contributions from the distinct parts involved in the interaction and, most importantly, to address some of the general questions posed above through a simple inverse modeling technique.

We start by considering a situation in which a NT and a FO are far apart. We focus on FO that are spatially localized and whose electronic structures are described by discrete energy levels. The system is described by a Hamiltonian $\hat{H}_{0}=\hat{h}_{\mathrm{NT}}+\hat{h}_{\mathrm{FO}}$, where $\hat{h}_{\mathrm{NT}}$ and $\hat{h}_{\mathrm{FO}}$ correspond to the individual Hamiltonians for the NT and the FO, respectively. Represented by a $\pi$-band nearestneighbour tight-binding Hamiltonian, $\hat{h}_{\mathrm{NT}}$ is written as $\hat{h}_{\mathrm{NT}}=\sum_{j, j^{\prime}}|j\rangle \gamma\left\langle j^{\prime}\right|$, where $|j\rangle$ is a Hilbert-space vector representing electronic states localized on the carbon atom labeled by the index $j$. This integer labels the atomic positions of the carbon atoms and consequently whether they are metallic or semi-conducting. The quantity $\gamma=2.66 \mathrm{eV}$ is the hopping integral for the carbon atoms of the pristine tube, and is hereafter chosen to be our energy unit. The electronic structure of the FO is represented by a single energy level, i.e., $\hat{h}_{\mathrm{FO}}=|\alpha\rangle \epsilon_{\alpha}\langle\alpha|$, where $|\alpha\rangle$ represents the electronic state localized at the FO and associated with a characteristic energy level $\epsilon_{\alpha}$. Despite the simplification, characterizing the FO by a single energy level can still describe the energy cost for extracting charge from it. We define the energy $\epsilon_{\alpha}$ as $\epsilon_{\alpha}=W-U_{\mathrm{I}}$, where $U_{\mathrm{I}}$ is the ionization potential of the $\mathrm{FO}$ and $W$ is the work function of the nanotube. This particular choice results from the fact that some amount of charge transfer often occurs when the two sub-systems are connected and which assumes that the individual vacuum levels of tube and adatom are aligned. The choice of $\hat{h}_{\mathrm{NT}}$ and $\hat{h}_{\mathrm{FO}}$ was primarily aimed at simplifying the notation and brings no limitation to its applicability. It is straightforward to generalize our method and lift both orbital and spin degeneracies as well as include more energy levels associated with the FO.

Both NT and FO have their individual electronic structures described by $\hat{h}_{\mathrm{NT}}$ and $\hat{h}_{\mathrm{FO}}$ but changes are expected when they are no longer in isolation. In this case, corrections to $\hat{H}_{0}$ should correspond to matrix elements spatially localized within the vicinity of the contact between tube and FO. We assume that the NT is efficient in screening local charge variations and that only the on-site energies of the carbon atoms nearest to the FO are to be affected, although screening clouds of variable sizes can also be used to account for imperfect screening. Likewise, the energy level of the FO is also allowed to change as a result of the proximity to the NT. Finally, the actual coupling between the parts is modelled by an added off-diagonal matrix element $\tau$ connecting the nearest sites on either side. In summary, the coupling between NT and FO is represented by the following perturbing potential:

$$
\hat{V}=|0\rangle \delta_{0}\langle 0|+| \alpha\rangle \delta_{\alpha}\langle\alpha|+| 0\rangle \tau\langle\alpha|+| \alpha\rangle \tau^{*}\langle 0|,
$$

where $\delta_{0}$ and $\delta_{\alpha}$ are the corrections to the on-site energies on the nearest carbon site (labeled $j=0$ ) and to the FO's energy level, respectively. Equation (1) corresponds to the situation in which a single carbon atom is the nearest NT site to the FO. This is, for instance, the case for $\mathrm{H}$ adatoms lying right above the nanotube atoms, which we refer to as a type-I FO. It is straightforward to generalize the expression above for when the FO lies closely to all six carbon atoms comprising a hexagon of the nanotube lattice. This is the case for several different adatoms whose most favourable location is above the centre of the nanotube hexagons [8], hereafter referred to as type II. In what follows, for the sake of brevity, we present a set of expressions for the type-I FO, knowing that it is very simple to rewrite them for their type-II counterpart [9].

The knowledge of the perturbing potential $\hat{V}$ fully determines the impact that the interaction with the FO has on the electronic structure of the system and consequently how its physical properties are affected, one of which is the conductance. This is most obviously done by expressing how the change in the global electronic density of states (DOS) depends on $\hat{V}$. This is defined as $\Delta \rho=\rho_{\mathrm{N} / \mathrm{F}}-\rho_{\mathrm{N}}-\rho_{\mathrm{F}}$, where $\rho_{\mathrm{N} / \mathrm{F}}$ is the DOS for the coupled system whereas $\rho_{\mathrm{N}}$ and $\rho_{\mathrm{F}}$ are the separate DOS for the NT and the FO, respectively. Based on the existence of special sum rules for the single-particle Green functions (GF) in the presence of localized perturbations, a tedious but straightforward derivation [10] leads to an expression for $\Delta \rho$ that reads

$$
\begin{aligned}
\Delta \rho(E)= & -\frac{1}{\pi} \operatorname{Im} \frac{\mathrm{d}}{\mathrm{d} E} \ln \left\{\left[1-\mathcal{G}_{0,0} \delta_{0}\right]\left[1-\mathcal{G}_{\alpha, \alpha} \delta_{\alpha}\right]\right. \\
& \left.\times\left[1-\frac{\mathcal{G}_{0,0} \mathcal{G}_{\alpha, \alpha}|\tau|^{2}}{\left(1-\mathcal{G}_{\alpha, \alpha} \delta_{\alpha}\right)\left(1-\mathcal{G}_{0,0} \delta_{0}\right)}\right]\right\},
\end{aligned}
$$

where $\mathcal{G}_{0,0}$ is the unperturbed GF for electrons of energy $E$ at the relevant NT site and $\mathcal{G}_{\alpha, \alpha}$ is the GF localized at the FO state $|\alpha\rangle$. One obvious advantage of eq. (2) is that it provides a transparent expression for the DOS change in terms of parameters clearly and distinguishably associated with the different parts of the system.

Most physical quantities depend directly on the DOS, which makes the equation for $\Delta \rho(E)$ particularly useful in providing information on how the properties of a NT 
are affected by the interaction with the FO. One such quantity is the total energy, whose difference between the coupled and uncoupled configurations gives the binding energy $\Delta \mathcal{E}$. It is defined as $\Delta \mathcal{E}=\mathcal{E}_{\mathrm{N} / \mathrm{F}}-\mathcal{E}_{\mathrm{N}}-\mathcal{E}_{\mathrm{F}}$, where $\mathcal{E}$ is the total energy and whose subscripts follow the same convention as used for the DOS. In the framework of the so-called tight-binding total-energy method, one can write the total energy $\mathcal{E}$ of a system as the electronic structure contribution added to a repulsive energy term [11], in which the latter has been given a formal correspondence with modern density functional theory [12]. This latter contribution is easily accounted for by including it as a small shift in the electronic on-site potential [11]. In this way, the total energy can be written as a sum over the eigenvalues of the Hamiltonian, which, in turn, allows us to express the binding energy as a function of $\Delta \rho$. It follows from a simple integration of eq. (2) that

$$
\begin{aligned}
\Delta \mathcal{E}= & \frac{1}{\pi} \int_{-\infty}^{E_{\mathrm{F}}} \mathrm{d} E \operatorname{Im} \ln \left\{\left[1-\mathcal{G}_{0,0} \delta_{0}\right]\left[1-\mathcal{G}_{\alpha, \alpha} \delta_{\alpha}\right]\right. \\
& \left.\times\left[1-\frac{\mathcal{G}_{0,0} \mathcal{G}_{\alpha, \alpha}|\tau|^{2}}{\left(1-\mathcal{G}_{\alpha, \alpha} \delta_{\alpha}\right)\left(1-\mathcal{G}_{0,0} \delta_{0}\right)}\right]\right\},
\end{aligned}
$$

where $E_{\mathrm{F}}$ is the Fermi level of the NT. Another representative quantity that reflects the strength of the interaction is the amount of charge exchanged between NT and FO. In terms of the parameters of the perturbing potential $\hat{V}$, the charge variation $\Delta \mathcal{C}$ on the $\mathrm{FO}$ is given by

$$
\begin{aligned}
\Delta \mathcal{C}= & -\frac{1}{\pi} \int_{-\infty}^{E_{\mathrm{F}}} \mathrm{d} E \\
& \times \operatorname{Im}\left\{\left[\left(\mathcal{G}_{\alpha, \alpha}\right)^{-1}-\delta_{\alpha}-\frac{\mathcal{G}_{0,0}|\tau|^{2}}{\left(1-\mathcal{G}_{0,0} \delta_{0}\right)}\right]^{-1}-\mathcal{G}_{\alpha, \alpha}\right\} .
\end{aligned}
$$

Equations (3) and (4) are closed-form expressions that relate the potential $\hat{V}$ with the binding energy and charge transfer, respectively, indicating that the knowledge of the former defines the latter two quantities. Alternatively, we can invert the equations so that the perturbation parameters are obtained from the values of $\Delta \mathcal{E}$ and $\Delta \mathcal{C}$. When combined with the fact that the total charge must be conserved, i.e., $\int_{-\infty}^{E_{\mathrm{F}}} \mathrm{d} E \Delta \rho(E)=0$, we have a set of equations that can uniquely determine $\hat{V}$. In other words, assuming that the binding energy and the charge transfer of a single FO attached to a carbon NT are known, we can derive the perturbing potential associated with this interaction. In fact, this has already been demonstrated by the authors for the case of adatoms [13] as FO. In that instance, the parameters $\delta_{0}, \delta_{\alpha}$ and $\tau$ were shown to be obtainable from $a b$ initio evaluations of $\Delta \mathcal{E}$ and $\Delta \mathcal{C}$.

Besides determining $\hat{V}$, here we want to explore another use for eqs. (3) and (4), namely, that they indicate the existence of a correlation between the binding energy and the charge transfer. For a given $\mathrm{NT} / \mathrm{FO}$ pair, $\Delta \mathcal{E}$ and
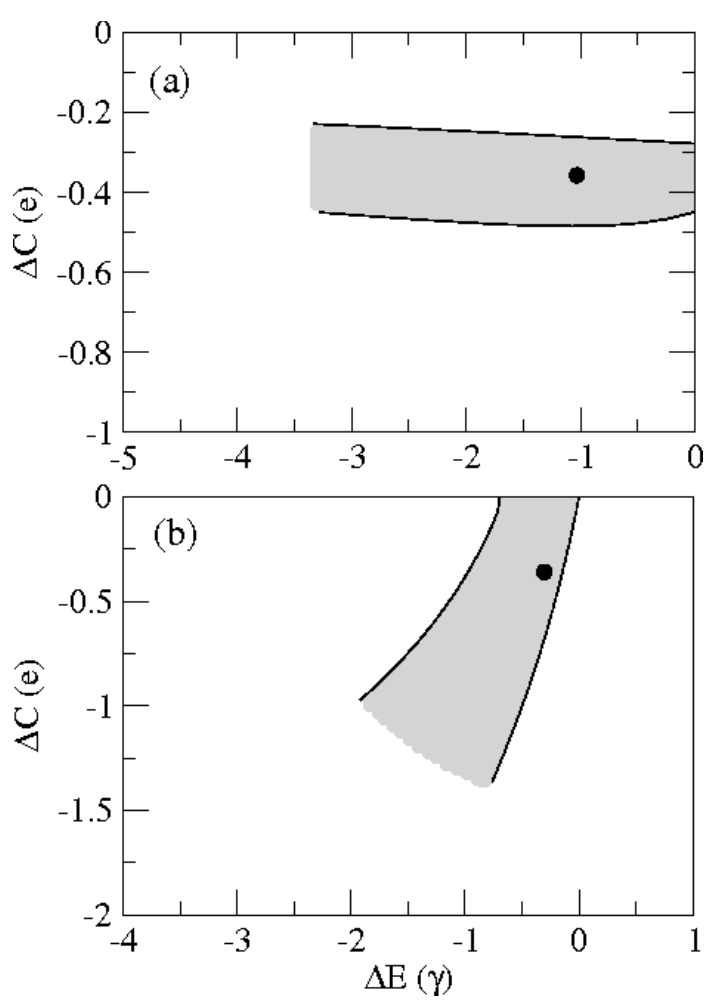

Fig. 1: $\Delta \mathcal{E} / \Delta \mathcal{C}$ graphs displaying the regions of allowed values for the binding energy and charge transfer for (a) a $\mathrm{H}$ adatom and (b) a Li adatom, both on a $(6,6)$ NT. In plotting fig. 1, the thick lines were obtained by selecting $\bar{\delta}_{0}=W$ and $\bar{\delta}_{0}=2 W$ on panel (a) and $\bar{\delta}_{0}=0$ and $\bar{\delta}_{0}=W / 12$ on panel (b). $\bar{\tau}=1.5 \gamma$ was used for both panels. The black dots represent the respective ab initio calculations.

$\Delta \mathcal{C}$ are not independent quantities but are constrained by their respective expressions above. The actual relationship between them depends on the specific values of the parameters $\delta_{0}$ and $\tau, \delta_{\alpha}$ being determined by demanding charge neutrality. If we were to choose those parameters arbitrarily, there is no guarantee that they would provide realistic figures for the binding energy and for the charge transfer. However, by varying the parameters up to carefully selected maximum values $\bar{\delta}_{0}$ and $\bar{\tau}$, one finds the corresponding range of possible values for both $\Delta \mathcal{E}$ and $\Delta \mathcal{C}$. We assume that the hopping element can vary as $\tau=0, \ldots, \gamma$ since, in general, this value cannot exceed the carbon-carbon hopping parameter. Concerning $\delta_{0}$ limits, we expect that the higher the local charge variation on the carbon atom connected to the impurity, the greater the correction to its energy level. We define a lower limit to $\delta_{0}$ when $\tau=0$ and for the maximum value, we assume that $\max \left\{\delta_{0}\right\}=2 \times \min \left\{\delta_{0}\right\}$. While this may be too broad a range, depending on the choice of $\bar{\delta}_{0}$ and $\bar{\tau}$, it highlights the relationship between those two quantities and, more importantly, points to an even broader range of incompatible combinations for binding energy and charge transfer. This is illustrated for the case of adatoms on a $(6,6)$ armchair NT, in which the gray regions of fig. 1 are shown as the likely ranges to contain the binding energies 
Table 1: List of FO with their values of $\epsilon_{\alpha}$, their respective types and the separately evaluated ab initio results for $\Delta \mathcal{E}$ and $\Delta \mathcal{C}$. The values of binding energy and charge transfer for a given NT/FO pair are predicted to fall within the following ranges: $\mathcal{L}^{-1}(\Delta \mathcal{C})>\Delta \mathcal{E}>\mathcal{U}^{-1}(\Delta \mathcal{C})$ and $\mathcal{U}(\Delta \mathcal{E})>\Delta \mathcal{C}>\mathcal{L}(\Delta \mathcal{E})$. The last column displays the DOS change for each FO. All results are for a $(6,6)$ armchair NT.

\begin{tabular}{|c|c|c|r|c|c|c|c|c|l|}
\hline FO & Type & $\epsilon_{\alpha}$ & \multicolumn{1}{c|}{$\Delta \mathcal{E}$} & $\Delta \mathcal{C}$ & $\mathcal{L}^{-1}(\Delta \mathcal{C})$ & $\mathcal{U}^{-1}(\Delta \mathcal{C})$ & $\mathcal{L}(\Delta \mathcal{E})$ & $\mathcal{U}(\Delta \mathcal{E})$ & $\frac{\Delta \rho\left(E_{\mathrm{F}}\right)}{\rho\left(E_{\mathrm{F}}\right)}$ \\
\hline $\mathrm{H}$ & I & -5.04 & -1.03 & -0.357 & - & - & -0.491 & -0.266 & 0.44 \\
$\mathrm{Xe}$ & I & -4.49 & 0.07 & -0.010 & 0.949 & -0.163 & -0.076 & 0.003 & 0.017 \\
$\mathrm{Ar}$ & $\mathrm{II}$ & -5.84 & 0.03 & -0.011 & 1.945 & -0.493 & -0.070 & 0.000 & 0.012 \\
$\mathrm{Li}$ & $\mathrm{II}$ & -1.99 & -0.30 & -0.360 & -0.134 & -0.973 & -0.693 & 1.095 & 1.86 \\
$\mathrm{Na}$ & $\mathrm{II}$ & -1.90 & -0.08 & -0.170 & -0.043 & -0.972 & -0.334 & 6.102 & 2.33 \\
$\mathrm{~N}_{2}$ & $\mathrm{II}$ & -5.77 & -0.01 & -0.011 & 1.901 & -0.407 & -0.038 & 0.031 & 0.097 \\
\hline
\end{tabular}

and charge transfers associated with those particular NT/FO pairs. The upper (lower) panel displays the results for a $\mathrm{H}(\mathrm{Li})$ adatom, which is a type-I (type-II) FO. The black dots, representing the corresponding ab initio evaluations of both $\Delta \mathcal{E}$ and $\Delta \mathcal{C}$, lie within the predicted range.

The gray regions of fig. 1 , hereafter referred to as $\Delta \mathcal{C} / \Delta \mathcal{E}$-diagrams, are delimited by lines whose equations are described by $\Delta \mathcal{C}_{u}=\mathcal{U}(\Delta \mathcal{E})$ and $\Delta \mathcal{C}_{\ell}=\mathcal{L}(\Delta \mathcal{E})$, where the functions $\mathcal{U}(x)$ and $\mathcal{L}(x)$ describe the upper and lower limit of the charge transfer, respectively. By knowing the functions $\mathcal{U}$ and $\mathcal{L}$ one can easily determine the whole range of possible values for $\Delta \mathcal{E}$ and $\Delta \mathcal{C}$. Furthermore, assuming either of the two quantities is known, possibly by an isolated measurement, one can use the functions $\mathcal{L}$ and $\mathcal{U}$ to predict the range of allowed values that the other quantity may have. For instance, if only the charge transfer $\Delta \mathcal{C}$ is known for a given NT/FO pair, the range of possible values for the corresponding binding energy is delimited by $\mathcal{L}^{-1}(\Delta \mathcal{C})$ and by $\mathcal{U}^{-1}(\Delta \mathcal{C})$, where $\mathcal{F}^{-1}$ is the inverse of function $\mathcal{F}$. Likewise, if only the binding energy $\Delta \mathcal{E}$ is known, the corresponding charge transfer is certain to fall within the range delimited by $\mathcal{L}(\Delta \mathcal{E})$ and $\mathcal{U}(\Delta \mathcal{E})$. Both $\mathcal{L}$ and $\mathcal{U}$ are polynomial functions of $\Delta \mathcal{E}$ described by $\Delta \mathcal{C}_{\ell}=\sum_{j} \ell_{j}(\Delta \mathcal{E})^{j}$ and $\Delta \mathcal{C}_{u}=\sum_{j} u_{j}(\Delta \mathcal{E})^{j}$ for the lower and upper limit, respectively, and that for the cases depicted in fig. 1 the non-zero coefficients $\ell_{j}$ and $u_{j}$ are $\ell_{0}=-0.28, \ell_{1}=-0.01, \ell_{2}=5.00 \times 10^{-4}, \ell_{3}=$ $2.00 \times 10^{-4}, u_{0}=-0.45, u_{1}=0.10, u_{2}=0.14, u_{3}=0.12$ on the upper panel and are $\ell_{0}=-0.02, \ell_{1}=2.43, \ell_{2}=0.88$, $\ell_{3}=0.80, u_{0}=7.78, u_{1}=10.58, u_{2}=5.30, u_{3}=1.27$ on the lower panel.

The results of fig. 1 are only valid for the specific $\mathrm{NT} / \mathrm{FO}$ pairs considered, i.e., $\mathrm{H}$ and $\mathrm{Li}$ adatoms on a $(6,6)$ NT. A different pair will lead to a different $\Delta \mathcal{C} / \Delta \mathcal{E}$-diagram but it is here that the mathematical transparency of eqs. (3) and (4) becomes really useful. Because both equations are given not only in terms of the $\hat{V}$-parameters but also in terms of $\mathcal{G}_{0,0}$ and $\mathcal{G}_{\alpha, \alpha}$, which carry specific information about the NT and the FO, respectively, changes in either component of the pair will have a predictable effect on the relationship between $\Delta \mathcal{E}$ and $\Delta \mathcal{C}$, and therefore on their corresponding diagram.
Put in another way, the functions $\mathcal{L}(\Delta \mathcal{E})$ and $\mathcal{U}(\Delta \mathcal{E})$ are described by polynomial equations whose coefficients depend on specific features of the NT/FO pair. By determining how the coefficients depend on those features, we can not only predict how the $\Delta \mathcal{C} / \Delta \mathcal{E}$-diagram changes but, more importantly, establish which features our pair must have to meet specific requirements regarding their binding energy and/or charge transfer.

To illustrate this point we assume the same NT as used in fig. 1 but this time with a variable $\epsilon_{\alpha}$. Bearing in mind our earlier definition, this corresponds to considering FO with different ionization potentials. The $\epsilon_{\alpha}$-dependence of the coefficients $\ell_{j}$ and $u_{j}$ indicates through the functions $\mathcal{L}$ and $\mathcal{U}$ how the $\Delta \mathcal{C} / \Delta \mathcal{E}$-diagram changes as different FO are considered. More specifically, by determining the coefficients $\ell_{j}\left(\epsilon_{\alpha}\right)$ and $u_{j}\left(\epsilon_{\alpha}\right)$, we are able to work out the respective functions $\mathcal{L}(\Delta \mathcal{E})$ and $\mathcal{U}(\Delta \mathcal{E})$, thus predicting the allowed range of values for their binding energy and charge transfer. It is natural to compare the predicted ranges with the actual values of binding energies and charge transfers obtained by separately evaluated $a b$ initio calculations. In addition to the $\mathrm{H}$ and $\mathrm{Li}$ adatoms already presented in fig. 1, a number of additional FO were considered, among which a variety of other adatoms as well as a few simple molecules. Table 1 shows those results for $\Delta \mathcal{E}$ and $\Delta \mathcal{C}$ associated with all the listed NT/FO pairs. It is remarkable that despite the simple description of the electronic structure of the separate parts, all results lie well within the predicted ranges.

At this point we are ready to answer some of the general questions posed earlier regarding the type of FO necessary to meet certain requirements. We can ask what features a FO must have to produce a specific set of binding energy $\Delta \mathcal{E}^{(0)}$ and charge transfer $\Delta \mathcal{C}^{(0)}$. The answer to such an inverse problem is now straightforward and follows from the solution of $\Delta \mathcal{C}^{(0)} \leqslant \mathcal{U}\left(\Delta \mathcal{E}^{(0)}\right)$ and $\Delta \mathcal{C}^{(0)} \geqslant \mathcal{L}\left(\Delta \mathcal{E}^{(0)}\right)$, which gives a range of possible values for $\epsilon_{\alpha}$. For instance, if one is looking for a FO on a $(6,6)$ NT for which the binding energy is around $\Delta \mathcal{E}^{(0)}=-0.16 \gamma$ and for $\Delta \mathcal{C}^{(0)}=-0.10 e$, our method suggests that good candidates are type-II FO whose ionization potentials are in the range $-1.24 \gamma>U_{\mathrm{I}}>-2.36 \gamma$. This range reduces the 
universe of possibilities one would have to try by the forward modeling approach. The values of $\Delta \mathcal{E}^{(0)}$ and $\Delta \mathcal{C}^{(0)}$ listed above correspond to those found in the case of a $\mathrm{K}$ adatom adsorbed to a $(6,6) \mathrm{NT}$. Being a type-II FO, the ionization potential $U_{\mathrm{I}}=-1.63 \gamma$ naturally meets the above requirements. Moreover, although we have varied the parameter $\epsilon_{\alpha}$ that characterizes the FO, we could also change the parameters defining the NT. In this case, either the diameter $d$ or the chiral angle $\theta$ of the NT host could be varied through the GF $\mathcal{G}_{0,0}$. A similar analysis would follow, this time providing a range of values for both $d$ and $\theta$ that meets the specific requirements of binding energy and charge transfer.

Finally, it is worth returning to the original goal of bridging the gap between the aforementioned stages involved in the calculations. Having written $\Delta \rho\left(E_{\mathrm{F}}\right)$, $\Delta \mathcal{E}$ and $\Delta \mathcal{C}$ under a common framework, these quantities are no longer evaluated in a disconnected fashion. Equations (2), (3) and (4) are able to relate the DOS change with the respective values of binding energy and charge transfer. Listed as a percentage of the original DOS, table 1 makes use of eq. (2) to express how $\Delta \rho\left(E_{\mathrm{F}}\right)$ is affected by the presence of each $\mathrm{FO}$, indicating $\mathrm{Na}$ as the one causing the largest variation. Bearing in mind that changes in the physical properties are intrinsically associated with changes in the DOS, this procedure indicates how effective a FO is in altering the NT transport properties, which, in turn, determines how good a sensor the NT/FO pair may turn out to be.

In summary, we have expressed the DOS variation $\Delta \rho\left(E_{\mathrm{F}}\right)$, the binding energy $\Delta \mathcal{E}$ and the charge transfer $\Delta \mathcal{C}$ that result from the interaction between a NT and a FO. Written in terms of a set of undetermined perturbation parameters, these expressions establish a constraint between those quantities, in particular the latter two. By varying the potential parameters up to carefully selected maximum values we are able to find a range of permitted values for both $\Delta \mathcal{E}$ and $\Delta \mathcal{C}$. Separately evaluated ab initio results for a number of FO lie within the predicted ranges. Furthermore, the mathematical transparency of our expressions allows us to distinguish the contributions coming from both parts involved in the interaction, enabling us to trace how the predicted range of values changes with variations of the NT/FO pair. This can be used to solve the inverse problem of finding FO meeting specific requirements on their binding energy and charge transfer. Our simple general approach can be used to provide guidelines in the search for ideal NT-based sensors.

\section{REFERENCES}

[1] Kong J., Franklin N. R., Zhou C., Chapline M. G., Peng S., Cho K. and Dai H., Science, 287 (2000) 622.

[2] Santucci S., Picozzi S., Di Gregorio F., Lozzi L., Cantalini C., Valentini L., Kenny J. M. and Delley B., J. Chem. Phys., 119 (2003) 10904.

[3] Snow E. S., Perkins F. K. and Robinson J. A., Chem. Soc. Rev., 35 (2006) 790.

[4] Charlier J. C., Blase X. and Roche S., Rev. Mod. Phys., 79 (2007) 677.

[5] Latil S., Roche S., Mayou D. and Charlier J. C., Phys. Rev. Lett., 92 (2004) 256805.

[6] Latil S., Roche S. and Charlier J. C., Nano Lett., 5 (2005) 2216.

[7] Durgun E., Dag S., Bagci V. M. K., Gulseren O., Yildirim T. and Ciraci S., Phys. Rev. B, 67 (2003) 201401

[8] ZhaO J., Buldum A., HaN J. and Lu J. P., Nanotechnology, 13 (2002) 195.

[9] Rocha C. G. and Ferreira M. S., in preparation.

[10] Zeller R., J. Phys.: Condens. Matter, 16 (2004) 6453.

[11] Cohen E. R., Mehl J. M. and Papaconstantopoulos A. D., Phys. Rev. B, 50 (1994) 14694.

[12] Foulkes W. M. C. and Haydock R., Phys. Rev. B, 39 (1989) 12520.

[13] Rocha C. G., Wall A., Rocha A. R. and Ferreira M. S., J. Phys.: Condens. Matter, 19 (2007) 346201. 